\title{
Proposed treatment for adolescent psychosis. 1: Schizophrenia and schizophrenia-like psychoses
}

\author{
Andrew F. Clark
}

Psychotic disorders in adolescence are uncommon, but often mark the beginning of a lifetime of contact with mental health services. They pose a challenge and anxiety to sufferers, to their families and to professionals involved in assessment and treatment. Accurate and early diagnosis, coupled with appropriate treatment, is essential to minimise secondary handicaps. However, their rarity means that professionals are frequently unfamiliar with some aspects of presentation or management and that appropriate service provision is not readily available (e.g. admission directly into an in-patient bed in an ageappropriate environment). A lack of age-appropriate provision may also mean that adult psychiatrists are occasionally called on to manage and treat a younger teenager presenting with a psychotic disorder.

The focus here will be on the assessment and treatment of first-onset psychotic disorder in adolescence and its subsequent longer-term management. Psychotic disorders solely due to substance misuse, or to other organic factors, will be considered in relation to the differential diagnosis and to acute treatment, but not specifically in relation to longerterm management. The longer-term management of psychotic disorders occurring as episodes as part of a bipolar affective disorder is the subject of separate review in a companion paper (Clark, 2001).

\section{Diagnostic criteria}

The majority of episodes of psychotic disorder in adolescence will be the first such episode of illness and there is therefore likely to be considerable diagnostic uncertainty, as the natural history of the disorder has yet to unfold (McClellan et al, 1993). It may be the first episode of a lifelong disorder such as one of the schizophrenias or a bipolar affective disorder; it may be a psychotic disorder secondary to other organic disorder (particularly substance misuse); or it may be a brief psychotic episode to be followed by full recovery with no further episodes of illness. Where possible, every attempt should be made to make a diagnosis within one of the standard diagnostic systems, ICD-10 or DSM-IV (World Health Organization, 1992; American Psychiatric Association, 1994). In most cases, the diagnostic criteria in adolescence are a little different from those in adulthood, albeit with a recognition that a lack of progress or development in a teenager may be the equivalent of deterioration in an adult. While there may be some reluctance to 'label' a young person with a diagnosis with potentially lifelong implications, such as schizophrenia or bipolar disorder, a diagnosis does have a number of advantages: it may act as a 'ticket of entry' (especially where services may be targeted towards 'the severely mentally ill') for the young person into resources or support not otherwise available; it may enable understanding and information-seeking by families; and it may behelpful in discussing the likely prognosis and future course of the illness. Hollis (2000), in his follow-up of adolescents admitted to the Maudsley Hospital with a diagnosis of psychosis, showed that the diagnostic stability of clear-cut first psychotic episodes meeting criteria for schizophrenia or for an affective psychosis is similar to that in adulthood. There will,

Andrew Clark has been Senior Lecturer in Adolescent Psychiatry in Manchester since 1997 (McGuinness Unit, Mental Health Services of Salford NHS Trust, Bury New Road, Prestwich, Manchester M25 3BL; e-mail: andrew.clark@man.ac.uk) and was previously Consultant in Adolescent Psychiatry within the West Midlands region from 1990 to 1997. He has particular interests in the assessment and treatment of young people with psychotic disorders. 
however, remain some individuals, particularly those with a mixed or schizoaffective presentation, in whom uncertainty will mean that the most appropriate diagnosis will simply be of a psychotic disorder, without any attempt at further specificity, and here the prognosis will necessarily need to be more guarded.

\section{Epidemiology}

There are few robust population data on incidence and prevalence of psychotic disorder in adolescence. Incidence increases markedly during the teenage years, with approximately a 50-fold difference between adulthood and childhood onsets (Beitchman, 1985). Gillberg et al (1986), in their Swedish population-based study, demonstrated a prevalence of $0.54 \%$ during the teenage years increasing from 0.9 per 10000 at age 13 to 17.6 per 10000 at age 18 . However, such figures mask the fact that in $5 \%$ of adult sufferers of schizophrenia the first episode has its onset before the 16th birthday (Asarnow \& Asarnow, 1994) and in 20\% before the age of 20 (Hafner \& Nowotny, 1995). All studies point to a preponderance of male over female sufferers, although this does vary with diagnostic criteria used and other methodological factors.

\section{Clinical presentation}

The signs and symptoms of a psychotic disorder differ little between disorders with their onset in adulthood and adolescence. However, the content of abnormal beliefs and perceptions are influenced by the individual's developmental stage, with preoccupations about bodily appearance or disfigurement, existential concerns and relationship concerns all common. In younger adolescents, the content may even relate to childhood themes of ghosts, monsters or film and TV characters. The onset may be acute or insidious, and in a number of cases there is a premorbid history of mild developmental or neurodevelopmental abnormality. In younger adolescents, social or intellectual deterioration may present itself as a failure to make progress and gain new skills rather than loss of old ones, and this is specifically recognised within DSM-IV criteria for schizophrenia.

\section{Prodromal schizophrenia?}

Some young people have a history of social gaucheness or oddness coupled with excessive sensitivity and magical thinking, but without core active symptoms of a psychotic nature. Some of these are referred to mental health services with the question "Might they be developing a psychotic disorder?" Currently, however, it appears impossible to differentiate with any degree of reliability those young people who will go on to develop a full-blown illness (the prodromal group) from those who will not. This makes initiating antipsychotic drug treatment contentious, as it would mean treating a significant number of people who may never develop the disorder and therefore confers minimal benefit and some risk. There is little option in this group, therefore, but to endeavour to monitor fairly closely and to try to ensure that they and their families have ready access to mental health services for early intervention, should more classical psychotic symptoms develop (Yung et al, 1996).

\section{Diagnostic assessment}

Full diagnostic assessment is essential in determining both treatment and likely course and prognosis of the disorder (McClellan \& Werry, 1994). A careful and detailed history should be obtained both from the young person and from his or her parents and / or carers. Unless a family is strongly opposed to it, information should also be sought from school or college, as they may have much useful information about changes in both cognitive and social functioning. Such contact will be a familiar practice to child and adolescent psychiatrists, but perhaps less so to adult psychiatrists, who will not necessarily be used to routine contact with employment settings.

Non-specific auditory hallucinations, coupled with a mistrust of others, may occur in a number of nonpsychotic conditions (e.g. conduct and emotional disorders, dissociative states, borderline personality disorders and post-traumatic stress disorder), and it is necessary to be clear about the nature of the phenomenlogy leading to a diagnosis of a psychotic disorder. Psychotic disorder secondary to an underlying physical illness is extremely rare but needs to be considered in every case. A formal physical examination, including a comprehensive neurological assessment, is therefore essential. The differential diagnosis is wide and encompasses many general medical conditions in addition to primary psychiatric disorders (e.g. epilepsies (particularly those with a temporal lobe focus), cerebral tumour or other space-occupying lesion and neurodegenerative disorders). Organic psychotic disorder secondary to substance misuse is frequently suspected in the teenage age group, and urine or hair analysis for illicit drugs should be undertaken. 
Box 1 Key issues in diagnostic assessment

Use of semi-structured interviews

Gathering of information from both young person and carer

Requesting permission for school/college contact, where applicable

Awareness of role and extent of physical investigation

Use of standard ICD-10 or DSM-IV diagnostic criteria

A positive result, however, merely indicates recent misuse, not causation, and it is necessary to observe the time course of the disorder in a drug-free environment before determining its significance. Comorbid substance misuse unrelated to the psychotic disorder is none the less an adverse prognostic factor and may require consideration and management in its own right.

In the absence of abnormal findings on physical examination, computed tomography scanning or magnetic resonance imaging are unlikely to detect any underlying neurological condition. However, they may still have a place in the management of the young person and his or her family in reassuring that all possibilities have been considered and explored. Other specialised investigations, such as of chromosomes, serum copper or autoantibodies, should be reserved only for when there are specific abnormalities or indications from the history or physical examination.

\section{Issues of consent and assent to treatment}

Ideally, the treating clinician will seek consent to treatment from both the young person and any adult holding parental responsibility for him or her. It may be that in younger teenagers it is the adult who gives informed consent, with the teenager assenting or acquiescing, while in older teenagers the consent is from the young person, with the parent supporting their decision. Explanations need to be tailored to the age and understanding of the young person, but should include an outline of the purpose, nature, likely effects and risks of any treatment proposed.

In some cases, however, there will be disagreement between young person and parent, or the parent alone will be giving consent. This poses both potential difficulties in longer-term compliance, where active collaboration between the sufferer and his or her family is often an important factor, but also major ethical and legal dilemmas around whether and how to mandate treatment. The issue of consent to treatment in a young person is complex and apparently contradictory. The legal systems within the UK differ between the principalities. In England and Wales, there is clear precedence that young people of sufficient age and understanding can give their own consent to treatment, even when an adult with parental responsibility for them is not giving consent. However, while this does mean that a clinician could start a willing 15 -year-old suffering from psychosis on antipsychotic medication, even in the face of parental opposition, there are other factors to consider. Allowing time for understanding and addressing the parent's concerns (see Family interventions below) may overcome their reluctance, but if they remain opposed to treatment, then discussion with a representative of the social services department of the local authority may be helpful in clarifying whether parental responsibility is being appropriately exercised.

Conversely, a young person's refusal of treatment does not overrule a parent consenting on his or her behalf. This could mean that a 16- or 17-year-old could be treated against his or her wishes solely on the basis of parental consent, although many would wish to consider the use of mental health legislation for the additional safeguards that it brings. Indeed, some would advocate consideration of its use in nonconsenting younger teenagers even where parental consent is forthcoming (there is no lower age limit to use of the Mental Health Act 1983 within England and Wales). Again, discussion with social work colleagues about the most appropriate course for this specific individual is usually helpful in establishing an agreed management plan. Further useful discussion of the legal and ethical dilemmas around consent to treatment in young people is to be found in Bailey \& Harbour (1998).

Box 2 Key issues in consent and assent

Assessment of competence and capacity to consent

Understanding of purpose, nature, likely effects and risks

Understanding of parental responsibility

Alternative legal mandates for treatment: parental consent; mental health act; children's legislation 


\section{Arena of treatment: in-, day or out-patient?}

One important aspect of management is determining the setting in which the young person can best be treated. Clearly, the prime consideration here has to be ensuring acceptable safety of the young person and of others, and some form of risk assessment process should be documented. Current or previous self-harm or violence towards others, particularly if associated with abnormal beliefs or perceptions, are pointers to a need for in-patient care. Lack of insight and likely poor compliance with investigation and assessments or suspicions of comorbid substance misuse as a causative or aggravating factor are also relative indications for admission. Weighed against these is the disruption that admission brings to the young person and his or her family, and that an age-appropriate setting may not be immediately available. Although admission to an in-patient adolescent unit may be the preferred option, often the only option available acutely is admission to an adult mental health ward. When this is the case, it should be supported where possible through secondment of nursing trained in working with adolescents.

Admission to an in-patient adolescent unit will permit an in-depth assessment and management plan to be started. This will usually include social and educational components and may also permit commencement of more intensive family intervention. In-patient approaches to treatment are discussed in greater detail in Cotgrove \& Gowers (1999). In other cases, however, it will be possible to assess and treat the young person as a day patient or even as an out-patient. This requires a relatively low level of risk of disturbed or aggressive behaviour and active engagement of the young person and his or her family. Such arrangements will need close and regular review, both of the young person's mental state and of the views of the family or carers.

Box 3 Key issues in choosing in-, day, or outpatient treatment

Risk to self or others

Psychosocial complexity

Availability of age-appropriate environment

Disruption of admission

Likely compliance and engagement with investigation and treatments
Close monitoring of risk to self and to others is essential, and it is wise already to have considered and developed contingency plans in case acute admission should become necessary. Premorbid social adjustment may be a better indicator of risk to others than the immediate psychopathology, although this merits further systematic study (Clare et al, 2000).

\section{Treatment planning and multi-modal intervention}

Treatment planning should address biological, psychological and social factors within a framework that also takes note of a young person's developmental stage. This will necessitate a combination or multi-modal approach to treatment that encompasses pharmacotherapy, individual psychological treatments, family-based interventions and educational or vocational strategies (McClellan \& Werry, 1994; Clark \& Lewis, 1998). Each of these needs careful consideration and explanation to the young person and his or her family. The emphasis on different forms of treatment varies through the course of the illness, with pharmacotherapy coupled with individual and family support emphasised in the early stages and psychological and vocational interventions predominating in the later stages.

\section{Pharmacological treatments}

The age-specific evidence-base for drug treatments of psychotic disorders in adolescence is very limited, and considerable reliance has to be placed on extrapolation from research among adult sufferers (Clark \& Lewis, 1998). In many cases, antipsychotic drugs shown to be effective in adults are not licensed for use in younger patients and this, and its meaning, should be explicitly discussed with families. Randomised controlled trials in children and adolescents have shown that antipsychotic medication is more effective than placebo (Spencer \& Campbell, 1994), that adverse effects are at least as common as in adult practice (Richardson et al, 1991) and that clozapine is effective in a proportion of otherwise treatment-resistant cases (Kumra et al, 1996). There is an increasing number of case reports of usage of the newer atypical agents, but these have not yet been formally evaluated. Nevertheless, there is a strong case to be made for their usage first-line, particularly in adolescent-onset psychosis. Early acceptable treatment with fewer adverse effects is likely to set up better patterns of treatment adherence and 
compliance and therefore to lead to more effective short- and long-term control of symptoms and therefore a better overall prognosis.

The choice of which atypical antipsychotic is probably determined largely by which side-effect is most undesirable in the particular patient. For example, for some the hazards of weight gain associated with olanzapine may be of greatest concern, while for others the muscle stiffness and other extrapyramidal motor effects more associated with risperidone may be those to be avoided. Dosages should be kept towards the lower end of the range where possible (e.g. $3 \mathrm{mg}$ daily risperidone; $10 \mathrm{mg}$ daily olanzapine) in order to minimise sideeffects. Treatment with any individual agent (assuming it is not producing unacceptable sideeffects) should be continued for 6-8 weeks, during which time mental state is monitored for signs of response. Partial response may indicate a need to increase the dosage, but little or no response by this point should prompt a change of drug.

If an individual fails to respond to two different antipsychotic drugs given in adequate dosage and over adequate duration, then the diagnosis and treatment compliance should both be reviewed. Covert substance misuse must be considered as a potential comorbid factor, and urine or hair analysis may be particularly illuminating. In cases where compliance with oral medication is a problem, and this cannot be overcome by compliance-enhancing strategies (e.g. education and discussion, motivational techniques, supervision of medication, oncedaily dosages, timing of administration, etc.), then it may be necessary to consider prescription of an older traditional neuroleptic in a depot preparation, notwithstanding their greater propensity for sideeffects. Assuming, however, that the diagnosis of a resistant psychotic disorder remains robust, then treatment with clozapine is the logical next step. This is now available only on an off-label and named-patient basis for young people aged under 16 years, and requires specific discussion with the pharmaceutical company's medical advisers in addition to the usual routine blood monitoring

Box 4 Key issues in pharmacological treatments

Limited age-specific evidence base Atypical $v$. typical antipsychotics Adequate dosages and durations Early consideration of clozapine Adjunctive benzodiazepine sedation Compliance enhancement strategies arrangement of the Clozaril Patient Monitoring Service.

In the acute phase, additional sedation may be necessary, either to relieve distress or to reduce disturbed or aggressive behaviour. The first choice should usually be of a benzodiazepine: either lorazepam 2-4 mg orally (or intramuscularly if the acuteness of the situation warrants it) or diazepam 5-10 mg orally. Neuroleptics such as haloperidol or droperidol should be reserved for those young people in whom benzodiazepines do not provide sufficient response.

The acute psychotic symptoms are likely largely to resolve over the initial $6-8$ weeks of treatment, but recovery obviously goes on much longer. Continued improvements are highly dependent on active rehabilitation through individual, family, social and educational or vocational interventions, but continued antipsychotic medication underpins this. There is little age-specific information upon which to base the decision of how long to continue medication, but 12-24 months post-resolution of acute symptoms does not seem unreasonable. Timing of reductions and withdrawal should be carefully considered and only undertaken at a time of relative stability in a young person's life. Re-emergence of symptoms on withdrawal will, of course, necessitate continuing medication longer.

However, some young people are likely to wish to stop medication sooner than this. This needs careful discussion with them and their carers about the increased risk of further episodes of illness and the adverse consequences on their lives of this. If it is not possible to persuade the young person to continue treatment for longer, then reduction and cessation should be as gradual as can be negotiated and should be followed by regular out-patient monitoring for some months post-cessation, if agreement to this can be obtained.

\section{Individual psychological approaches}

Establishment of a supportive psychotherapeutic relationship with the young person is the second key component of treatment. Initially, the focus of this is likely to be one of coming to terms with the illness, its treatment and its effects on the young person and his or her family. Psychotic experiences can be both puzzling and frightening for the sufferer, and explanation and reassurance will be necessary. Concerns about the nature of the illness and its treatment are likely to need addressing recurrently. Time for questions about prognosis and effects on schooling, relationships and career plans is 
important, and there may be bereavement-like issues of loss to be addressed. More specific individual psychological therapies may also be helpful in later stages of the illness, when social skills training may be a part of a rehabilitation package, or when cognitive-behavioural strategies might be employed in either compliance enhancement or in developing better control of residual symptoms (Penn \& Mueser, 1996; Scott, 1999). These have not as yet been formally evaluated in adolescents, but results from studies among adult sufferers appear promising.

\section{Family interventions}

It is impossible to consider young people separately from their families. In younger teenagers, the active recruitment and engagement of parents into the treatment plan are essential, and considerable time may be needed in explanation and discussion of the illness and its treatments. The place of family interventions in the management of adults suffering from schizophrenia is now well established and has been clearly demonstrated to reduce short- and longerterm relapse (de Jesus Mari \& Streiner, 1994). Such interventions classically focus on giving information about the illness, its effects and its treatment and on reducing higher levels of hostile or critical expressed emotion within the family. These interventions have not been formally evaluated in families of adolescent patients, and there are dangers in direct extrapolation of their findings (Hollis, 2000), especially as parents of children and adolescents with psychosis show lower levels of expressed emotion than do parents of adult sufferers (Asarnow et al, 1994). However, this does not mean that reduction of high expressed emotion, where it does exist, should not be a specific treatment goal. In young people, there are also major bereavement and loss issues for parents around the child's lost or damaged adulthood, particularly if the sufferer is left with major residual disabilities or impairments. Clarifying what is appropriate parental concern and what is parental over-protection in these instances may need considerable discussion and time. Many families will also include siblings, who may merit support and information in their own right as they struggle with the changes in their brother or sister, the preoccupations of their parents and their own anxieties for their own mental health. Other potential avenues of support for parents and siblings are from within the voluntary sector through carers' groups, such as those run by the National Schizophrenia Fellowship or Mind, and it is useful to be able to give families local contact telephone numbers or addresses for these.

\section{Educational and vocational approaches}

Adolescence is a time of considerable social and educational transition. Previous educational and career plans are likely to have been affected by the young person's illness, and the implications of this need active management. This liaison with other agencies should be started early in the course of the illness episode, even before the eventual outcome is clear. It is better to have these additional services available, even if it turns out that they are not needed.

The young person and his or her family are likely to each have additional needs consequent on the illness. There should be joint assessment of need by health and social services in accordance with a Care Programme Approach. Often, a local authority social services department may need to be encouraged to draw on expertise from within both its child care teams and its mental health teams, as it is relatively rare to find an individual social worker with experience of both.

Where a young person is still attending school, early contact with the school, and if necessary the special needs coordinator, can help pave the way for return or, equally important, identify that return is unlikely to be viable. It may then be important to involve the educational psychologist about more specific educational provision. For older teenagers, the option of a supported training placement within a further education college should be considered. For some young people, a specialised residential placement, such as those provided by the Richmond Fellowship or Making Spaces, may better offer the intensive combination of educational, psychological and social approaches that their rehabilitation needs demand.

\section{Outcome, prognosis and longer-term planning}

Outcome studies of adolescent-onset psychosis are limited and selective in nature, making interpretation difficult. Gillberg et al (1993), Cawthron et al (1994) and Hollis (2000) each report long-term follow-up studies of young people admitted to inpatient units in Sweden, Oxford and the Maudsley, respectively. All studies demonstrate a majority of cases having a poor prognosis, with recurrent illness and markedly impaired social functioning. There was a tendency for those individuals with more significant affective symptomatology to do slightly 
better, but this was not consistent. These findings are in keeping with clinical experience, although it is possible that the advent of the atypical antipsychotics may improve prognosis through better early compliance and thereby an overall reduced period of acute illness (Wyatt, 1995).

A few young people will have a single psychotic episode, make a full recovery and be treated solely within child and adolescent mental health services. These are very much the minority, and in most cases a transition into adult mental health services within a Care Programme Approach will be necessary. This transition needs careful planning and management, both among the agencies and with the young person and the family. Wherever possible, a series of graded minor transitions should be planned, so that there is always one professional worker with whom the young person and family feel they have an ongoing relationship and who is familiar with their care and needs and can therefore act as a point of continuity for them and for other professional workers involved.

\section{References}

American Psychiatric Association (1994) Diagnostic and Statistical Manual of Mental Disorders, 4th edn (DSM-IV). Washington, DC: APA.

Asarnow, J. R., Tompson, M., Hamilton, E. B., et al (1994) Family expressed emotion, childhood-onset depression, and childhood-onset schizophrenia spectrum disorders: is expressed emotion a non-specific correlate of child psychopathology or a specific risk factor for depression? Journal of Abnormal Child Psychology, 22, 129-146.

Asarnow, R. F. \& Asarnow, J. R. (1994) Childhood-onset schizophrenia: editors' introduction. Schizophrenia Bulletin 20, 591-597.

*Bailey, S. \& Harbour, A. (1998) The law and a child's consent to treatment (England and Wales). Child Psychology and Psychiatry Review, 4, 1-5.

Beitchman, J. H. (1985) Childhood schizophrenia: a review and comparison with adult-onset schizophrenia. Psychiatric Clinics of North America, 8, 793-814.

Cawthron, P., James, A., Dell, J. et al (1994) Adolescent onset psychosis. A clinical and outcome study. Journal of Child Psychology and Psychiatry, 35, 1321-1332.

Clare, P., Bailey, S. \& Clark, A. (2000) Relationship between psychotic disorders in adolescence and criminally violent behaviour: a retrospective examination. British Journal of Psychiatry, 177, 275-279.

Clark, A. F. (2001) Bipolar illness in adolescents. Advances in Psychiatric Treatment, 8, in press.

* — \& Lewis, S. W. (1998) Practitioner review: treatment of schizophrenia in childhood and adolescence. Journal of Child Psychology and Psychiatry, 39, 1071-1081.

${ }^{*}$ Cotgrove, A. J. \& Gowers, S. G. (1999) Use of an adolescent in-patient unit. Advances in Psychiatric Treatment, 5, 192 199.

de Jesus Mari, J. \& Streiner, D. L. (1994) An overview of family interventions and relapse on schizophrenia: metaanalysis of research findings. Psychological Medicine, 24, 565-578.

Gillberg, C., Wahlstrom, J., Forsman, A., et al (1986) Teenage psychoses - epidemiology, classification and reduced optimality in the pre-, peri- and neonatal periods. Journal of Child Psychology and Psychiatry, 27, 87-98.

Gillberg, I. C., Hellgren, L. \& Gillberg, C. (1993) Psychotic disorders diagnosed in adolescence. Outcome at 30 years. Journal of Child Psychology and Psychiatry, 34, 1173-1186.

Hafner, H. \& Nowotny, B. (1995) Epidemiology of early onset schizophrenia. European Archives of Psychiatry and Clinical Neurosciences, 245, 80-92.

Hollis, C. (2000) Adolescent schizophrenia. Advances in Psychiatric Treatment, 6, 83-92.

Kumra, S., Frazier, J. A., Jacobsen, L. K., et al (1996) Childhood onset schizophrenia: a double blind clozapine haloperidol comparison. Archives of General Psychiatry, 53, 1090-1097.

McClellan, J. M., Werry, J. S. \& Ham, M. (1993) A follow-up study of early onset psychosis: comparison between outcome diagnoses of schizophrenia, mood disorders and personality disorders. Journal of Autism and Developmental Disorders, 23, 243-262.

* — \& - (1994) Practice parameters for the assessment and treatment of children and adolescents with schizophrenia. Journal of the American Academy of Child and Adolescent Psychiatry, 33, 616-635.

Penn, D. L. \& Mueser, K. T. (1996) Research update on the psychosocial treatment of schizophrenia. American Journal of Psychiatry, 153, 607-617.

Richardson, M. A., Haughland, G. \& Craig, T. J. (1991) Neuroleptic use, parkinsonian symptoms, tardive dyskinesia and associated factors in child and adolescent psychiatric patients. American Journal of Psychiatry, 148, 1322-1328.

Scott, J. (1999) Cognitive and behavioural approaches to medication adherence. Advances in Psychiatric Treatment, 5, 338-345.

Spencer, E. K. \& Campbell, M. (1994) Children with schizophrenia: diagnosis, phenomenology, and pharmacotherapy. Schizophrenia Bulletin, 20, 713-725.

World Health Organization (1992) The ICD-10 Classification of Mental and Behavioural Disorders: Clinical Descriptions and Diagnostic Guidelines. Geneva: WHO.

Wyatt, R. J. (1995) Early intervention in schizophrenia: can the course of the illness be altered? Biological Psychiatry, $38,1-3$.

Yung, A. R., McGorry, P. D., McFarlane, C. A., et al (1996) Monitoring and care of young people at incipient risk of psychosis. Schizophrenia Bulletin, 22, 283-303.

* indicates articles of particular interest

\section{Multiple choice questions}

1. Assessment of an adolescent with possible psychosis should routinely include:

a urinary or hair drug screen

b parental interview

c school or college report

d chromosomal analysis

e MRI scanning.

2. In treatment of adolescents with schizophrenia: a parents may give consent on behalf of a young person

b parents are likely to show high expressed emotion

c family interventions are contraindicated 
$d$ the needs of siblings should also be considered

e the effectiveness of family interventions has not been formally evaluated.

3. Antipsychotic drug treatment in adolescent schizophrenia:

a is rarely necessary

b may include use of clozapine in otherwise treatment-resistant cases

$c$ is not supported by any age-specific randomised controlled trial evidence

d should be only a part of a more comprehensive management plan

e should be started only if the young person is an in-patient.

4. Schizophrenia in adolescence:

a usually has a good outcome

$\mathrm{b}$ is not possible to diagnose accurately

c has a better prognosis when there is also comorbid substance misuse d can be accurately recognised in its prodromal phase

e may sometimes be successfully treated solely on an out-patient basis.
MCQ answers

$\begin{array}{lllll}\text { 1 } & \text { 2 } & 3 & 4 \\ \text { a T } & \text { a T } & \text { a F } & \text { a F } \\ \text { b T } & \text { b F } & \text { b T } & \text { b F } \\ \text { c T } & \text { c F } & \text { c F } & \text { c F } \\ \text { d F } & \text { d T } & \text { d T } & \text { d F } \\ \text { e F } & \text { e T } & \text { e F } & \text { e T }\end{array}$

\section{Advances in Psychiatric Treatment}

Print (plus FREE on-line)

$\begin{array}{llllllll} & \text { Member } & \text { Individual } & \text { Institution } & & \text { Member } & \text { Individual } & \text { Institution } \\ & & & & \text { Worldwide } & £ 30 / \$ 48 & £ 68 / \$ 109 & £ 90 / \$ 44 \\ \text { Europe } & £ 40 & £ 88 & £ 99 & & & \\ \text { USA } & \$ 75 & \$ 156 & \$ 174 & & & \\ \text { ROW } & £ 46 & £ 95 & £ 106 & & \end{array}$

On-line (only)

Airmail at the addition cost of $£ 18 / \$ 29$

For more information and to order a subscription please contact:

Royal Society of Medicine, Subscriptions Department, PO Box 9002, London, W1A OZA, UK

Telephone +44 (0)20 7290 2927; fax +44 (0)20 72902929.

\section{www.rcpsych.org}

